\title{
Métodos de Avaliação do Desempenho de Redes Ópticas Analisando as Curvas de Bloqueio
}

\author{
Yruí G. Neris ${ }^{1}$, Rodrigo S. Tessinari ${ }^{2}$, Débora C. F. Lopes ${ }^{1}$, Anilton S. Garcia ${ }^{1}$, \\ Claunir Pavan ${ }^{3}$, Marcia H. M. Paiva ${ }^{1}$ \\ ${ }^{1}$ Laboratório de Telecomunicações - Universidade Federal do Espírito Santo (UFES) \\ Vitória - ES - Brasil \\ ${ }^{2}$ University of Bristol \\ Bristol - United Kingdom \\ ${ }^{3}$ Ciência da Computação - Universidade Federal da Fronteira Sul (UFFS) \\ Chapecó - SC - Brasil \\ \{yrui.neris, debora.lopes\}@aluno.ufes.br, rodrigo.tessinari@bristol.ac.uk \\ anilton@inf.ufes.br, claunir.pavan@uffs.edu.br, marcia.paiva@ufes.br
}

\begin{abstract}
The continuous change in the scenario of traffic demands requires implementation of traffic engineering techniques to increase the efficiency of resource utilization. Therefore, it is important to implement methods to evaluate the results of the proposed techniques in order to assist decision-making processes, especially in scenarios where it is not possible to identify the best results visually. In this work two methods are proposed to evaluate the performance of optical networks from the blocking rate results. The methods evaluate the results for different traffic loads and demonstrate that it is possible to identify the networks that perform better based on the blocking curves.
\end{abstract}

Resumo. A alteração contínua no cenário das demandas de tráfego nas redes de telecomunicações exige a implementação de técnicas de engenharia de tráfego e rede, que permitam aumentar a eficiência da utilização dos recursos. Portanto, é importante a implementação de métodos que sejam capazes de avaliar os resultados das técnicas propostas, a fim de auxiliar nas tomadas de decisão, especialmente em cenários onde não é possível identificar os melhores resultados visualmente. Neste trabalho são propostos dois métodos para avaliar o desempenho de redes ópticas a partir dos resultados de taxa de bloqueio. Os métodos avaliam os resultados para diferentes cargas de tráfego, e demonstram que é possivel identificar as redes que apresentam melhor desempenho com base nas curvas de bloqueio.

\section{Introdução}

A popularização da internet e a necessidade de trocar informações aumentou a importância das redes de telecomunicações e a demanda do tráfego de dados. A tendência é que o tráfego de dados continue aumentando nos próximos anos, chegando a 20,2 Exabytes por mês em 2020 [Chatterjee et al. 2018]. Além disso, o caráter dinâmico do tráfego atual exige, para otimização da infraestrutura, a implementação de técnicas de engenharia de tráfego e de projeto de redes, a fim de otimizar os recursos consumidos. 
Atualmente a maioria das redes de telecomunicações faz uso da transmissão baseada em Multiplexação por Divisão em Comprimentos de Onda (WDM), em que os canais possuem espaçamento fixo de $50 \mathrm{GHz}$ ou $100 \mathrm{GHz}$. Esses canais são compatíveis com requisições de até $100 \mathrm{~Gb} / \mathrm{s}$ [Chatterjee et al. 2015]. Contudo, já há demanda por canais de transmissão na faixa dos terabits por segundo, o que motiva o desenvolvimento de novas tecnologias e arquiteturas de rede. As redes ópticas elásticas (EON), promissoras para novas instalações, prometem suportar canais de até $1 \mathrm{~Tb} / \mathrm{s}$ em função da flexibilidade do espaçamento entre os canais [Gerstel et al. 2012].

Na Figura 1 é apresentada uma grade com espaçamento fixo, de $50 \mathrm{GHz}$ e uma grade flexível, onde é possível identificar a ineficiência da utilização do espectro da rede de grade fixa quando comparada com a rede de grade flexível. Na rede de grade fixa, é possível notar que canais com taxas de dados inferiores a $100 \mathrm{~Gb} / \mathrm{s}$ geram desperdício de recursos já que canais de $10 \mathrm{~Gb} / \mathrm{s}$ e $100 \mathrm{~Gb} / \mathrm{s}$ consomem a mesma largura de banda. Por outro lado, as redes com grade flexível possibilitam a transmissão em taxas mais elevadas, além da utilização do espectro de forma mais eficiente, proporcionando uma maior flexibilidade para aprovisionamento de novos serviços. Esta condição ocorre devido à capacidade de agregar múltiplas subportadoras e dividir o espectro em granularidade menor, ex. 12,5 GHz [Gerstel et al. 2012].

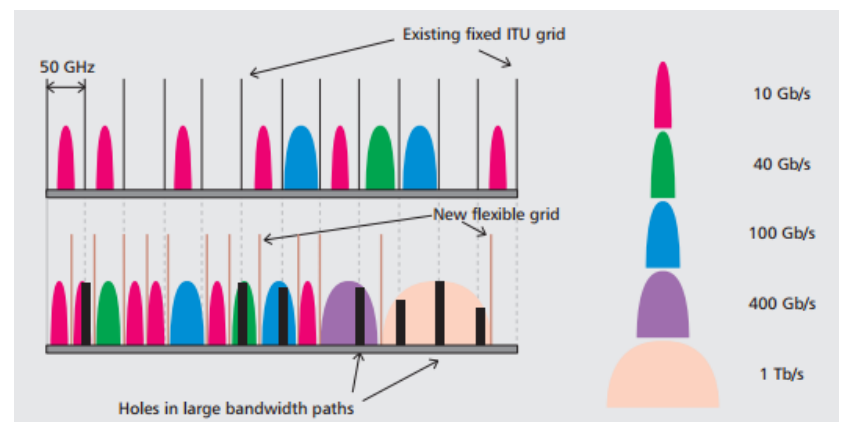

Figura 1. Comparação entre grade fixa e flexível para várias taxas de bit [Gerstel et al. 2012].

Com o advento das redes elásticas surgem novos desafios como o problema de Roteamento e Atribuição de Espectro (RSA) [Chatterjee et al. 2015] que consiste em encontrar um caminho para cada requisição, alocando o número de slots necessários para atender à requisição solicitada ao longo do caminho. A janela de espectro possui um tamanho finito e a técnica usada para resolver o problema do RSA tem influência na taxa de bloqueio de requisições que não podem ser alocadas em função da falta de recursos disponíveis para a alocação no espectro ou outras restrições impostas pela tecnologia.

Nos trabalhos [Tessinari et al. 2018b], [Zang et al. 2000], [Tessinari et al. 2015] e [Tessinari et al. 2018a] é possível verificar que os resultados demonstrados através de curvas de bloqueio variam conforme as cargas da rede. Por exemplo, em [Tessinari et al. 2018b] é demonstrada a influência que a topologia causa no desempenho de redes ópticas, onde topologias com a mesma quantidade de recursos possuem diferentes taxas de bloqueio, e no trabalho [Zang et al. 2000] é possível verificar como os resultados de bloqueio variam conforme as cargas da rede para diferentes heurísticas de atribuição de comprimento de onda. Nesses cenários, quando analisadas em conjunto todas as curvas de bloqueio geradas a partir de simulações, é possível identificar que uma 
determinada curva possui o menor bloqueio para uma dada carga de rede e para outra carga apresenta o maior bloqueio dentre todas as curvas do conjunto analisado. Com isso, observa-se a necessidade de métodos que auxiliem na identificação dos melhores resultados apresentados através das curvas de bloqueio em uma análise global, ou seja, de identificar o melhor resultado comparando todas cargas de rede dentro de um intervalo.

Tendo em vista essa característica, neste trabalho são propostos dois métodos para avaliação dos resultados das curvas de bloqueio provenientes de simulações de roteamento em redes de telecomunicações. Um método avalia os resultados analisando os valores da curva para cada carga de rede e o outro avalia a área sob a curva. Os métodos possuem o objetivo de identificar o resultado que representa o melhor desempenho dentro do intervalo analisado. Os resultados de bloqueio são gerados a partir da análise das taxas de bloqueio obtidas através da aplicação do RSA em um conjunto de topologias com a mesma quantidade de recursos. A partir dos resultados obtidos, não é possível identificar, de uma forma visual, a topologia que possui um melhor desempenho em uma análise global. Nesse caso, dizer que uma topologia possui melhor desempenho que as demais, é dizer que ela possui menor taxa de bloqueio.

Este trabalho está organizado da seguinte forma: Na Seção 2 é apresentado, resumidamente, o problema de RSA. Na Seção 3 são apresentados a motivação e os métodos desenvolvidos para a avaliação dos resultados da taxa de bloqueio e, finalizando, os resultados obtidos e as conclusões são apresentadas nas Seções 4 e 5, respectivamente.

\section{Problema de Roteamento e Alocação de Espectro}

As redes ópticas elásticas possibilitam a utilização do espectro de forma eficiente, aumentando a capacidade de escoamento de tráfego. Os algoritmos de RSA são utilizados no cálculo de caminhos e na alocação de espectro, adequados para atender requisições na rede, obedecendo as restrições de continuidade e contiguidade de espectro.

Nas redes com grade fixa, a alocação do espectro possui a restrição de continuidade de comprimento de onda. O slot que atende a requisição deve possuir o mesmo índice em todos os enlaces ao longo do caminho. Nas redes com grade flexível, além da restrição da continuidade de espectro, análoga à restrição das redes com grade fixa, existe também a restrição de contiguidade de espectro. Caso uma requisição necessite de mais de um slot, estes devem ser adjacentes [Chatterjee et al. 2015].

Utilizando a teoria de grafos para definir uma topologia, a rede pode ser representada por um grafo $G=(V, E)$ onde $V$ é o conjunto de vértices que representam os nós da rede e $E$ é o conjunto de arestas que representam os enlaces bidirecionais. A Figura 2 ilustra as restrições de continuidade e contiguidade de espectro em redes ópticas elásticas para uma rede de 4 nós e 4 enlaces bidirecionais. Considerando uma requisição entre os nós 1 e 4, que necessite 2 slots, temos dois caminhos possíveis, 1-2-4 e 1-2-3-4. Contudo, note que o primeiro caminho fere a restrição de continuidade, uma vez que os enlaces 1-2 (enlace 1) e 2-4 (enlace 4) possuem 2 slots contíguos, porém eles não são contínuos ao longo do caminho. Neste caso a requisição será bloqueada Figura 2 (a). O caminho 1-2-34 respeita as restrições de continuidade e contiguidade, sendo possível alocar a requisição nos slots 5 e 6 em todos os enlaces do caminho Figura 2 (b).

O problema do RSA é de complexidade NP-Difícil [Wang et al. 2011] e pode ser simplificado em dois subproblemas, (i) subproblema de roteamento e (ii) subproblema de 


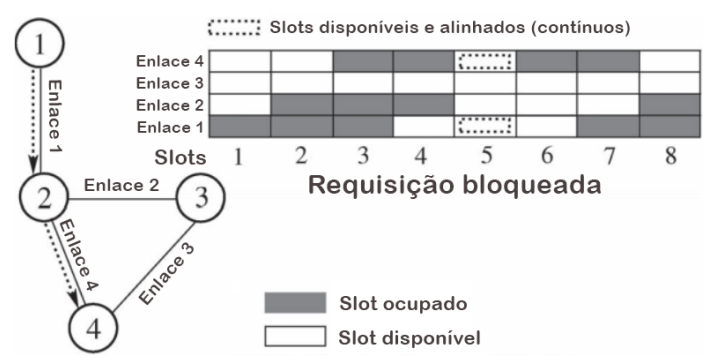

(a)

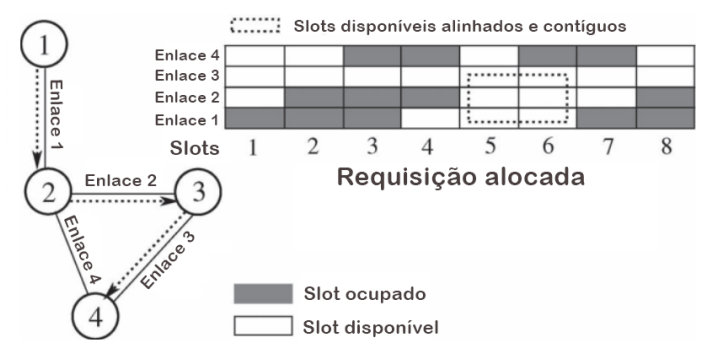

(b)

Figura 2. (a) Exemplo de bloqueio de requisição devido a Restrição de Continuidade e Contiguidade. (b) Alocação de requisição atendendo a Restrição de Continuidade e Contiguidade [Chatterjee et al. 2015].

alocação de espectro [Chatterjee et al. 2015]. As técnicas de roteamento e alocação de espectro utilizadas neste trabalho são apresentadas a seguir.

\subsection{Roteamento e Alocação de Espectro}

Existem diferentes métodos de roteamento e alocação de espectro [Chatterjee et al. 2015] [Chatterjee et al. 2018]. Neste trabalho são utilizadas as políticas de roteamento fixo [Ramamurthy and Mukherjee 2002], e a alocação de espectro firstfit [Rosa et al. 2012].

- Roteamento Fixo (FR): Uma única rota é pré-computada para cada par de nós origem-destino usando o caminho mínimo (em número de enlaces) que, é encontrado com a utilização do algoritmo de Dijkstra [Dijkstra 1959]. Quando o caminho é selecionado, verifica-se a disponibilidade de slots em quantidade suficiente e que atendam as restrições, seguindo a política de alocação. Caso não houver disponibilidade a requisição é bloqueada. [Chatterjee et al. 2015].

- First-Fit (FF): Nessa política de alocação os slots são indexados em ordem crescente e é mantida uma lista com os slots ocupados e livres no espectro. Para toda alocação, são escolhidos os slots com os menores índices que satisfaçam as restrições de continuidade e contiguidade em todos os enlaces do caminho a ser alocado. A política FF não requer informações globais da rede e a forma de escolha de slots proporciona um maior espaço contíguo nos enlaces da rede. A complexidade de implementação desta política é baixa e é reconhecida como adequada para a alocação de espectro [Chatterjee et al. 2018].

\section{Métodos para avaliação das curvas de bloqueio}

A topologia de uma rede de telecomunicações influencia seu desempenho tanto em termos de Probabilidade de Bloqueio e Throughput [Tessinari et al. 2018b], como em termos de sobrevivência [Pavan et al. 2015], e modificar a topologia afeta diretamente o seu desempenho. Redes com a mesma quantidade de recursos podem ter taxas de bloqueios diferentes para a mesma carga de tráfego.

A Figura 3 exibe o resultado das taxas de bloqueio de cinco topologias, geradas adicionando um enlace à topologia de 22 nós e 31 enlaces da Rede Nacional de Ensino e Pesquisa [RNP 2015]. Dessa forma, a única diferença entre as 5 topologias é relativa 
ao enlace adicional. Para cada topologia, foram realizadas simulações com nove cargas de tráfego, sendo possível observar que todas possuem um melhor desempenho, se comparado com as demais, para uma carga da rede específica, dificultando a identificação da topologia com o melhor desempenho em um contexto global.

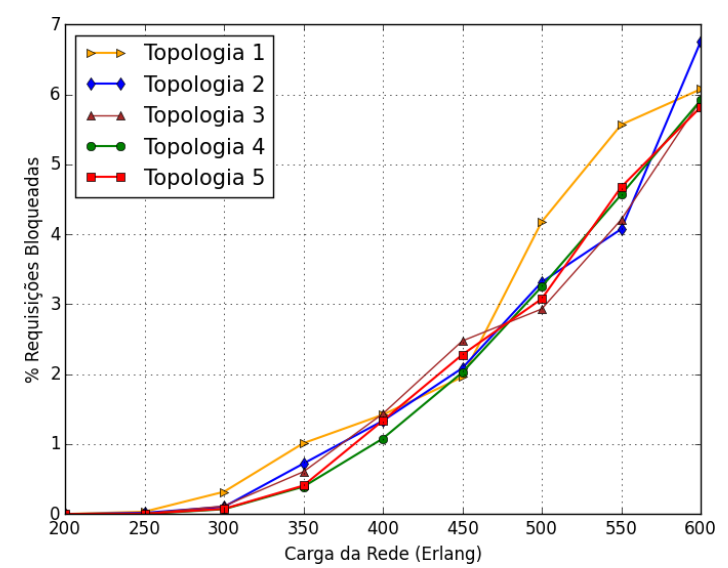

Figura 3. Curvas de bloqueio de 5 topologias diferentes.

Atualmente, não foram encontrados, no esforço de pesquisa deste trabalho, estudos que se propõem a realizar a análise do desempenho de um conjunto de redes para o cenário apresentado, onde o desempenho varia conforme a carga da rede. Tendo em vista essa característica, e a ausência de estudos que abordam esse cenário, são apresentados neste artigo dois métodos de avaliação das curvas de bloqueio obtidas através da aplicação do algoritmo de RSA, para a identificação da topologia com o melhor desempenho, em um contexto global. Ambos os métodos avaliam as curvas de bloqueio em todas as cargas da rede, independentemente dos cenários a partir dos quais elas foram geradas, gerando um valor que representa o desempenho para cada curva, e esse valor representa o desempenho da topologia.

Para ambos os métodos, primeiramente é aplicado um filtro, limitando o bloqueio máximo permitido utilizando o cálculo de percentil, onde as topologias que tiverem um bloqueio superior ao permitido para qualquer carga da rede, são desclassificadas.

Para exemplificação utilizamos a Figura 4 (a), onde se tem o limite de bloqueio estabelecido arbitrariamente com um percentil de $80 \%$, considerando somente as topologias que em todas as cargas de rede analisadas estiveram entre as 4 melhores ( $80 \%$ de 5) topologias.

Para cada carga da rede o valor do limite máximo de bloqueio permitido é o valor correspondente ao valor da quarta (80\% de 5) topologia com o menor bloqueio, e a topologia que possuir um valor superior é desclassificada. Para o caso da Figura 4 (a) as topologias 1, 2 e 3 são desclassificadas, já que a topologia 1 possui um bloqueio maior que o limite permitido nas cargas de rede 300, 350, 500 e 550, a topologia 2 na carga 600 e a topologia 3 na carga 450 . Restam, portanto, apenas as topologias 4 e 5, exibidas na Figura 4 (b). O objetivo do filtro é manter a topologia que, comparada com as demais, teve um comportamento mais estável, se mantendo entre as melhores, não apresentando um resultado considerado ruim para alguma carga da rede.

Após a aplicação do filtro, as curvas classificadas são avaliadas em todas as cargas 


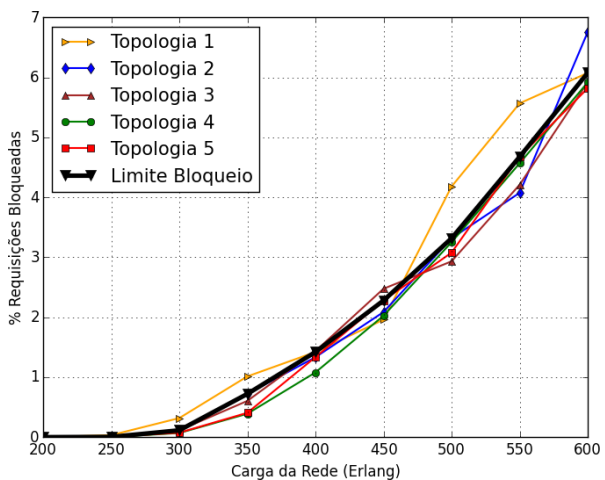

(a)

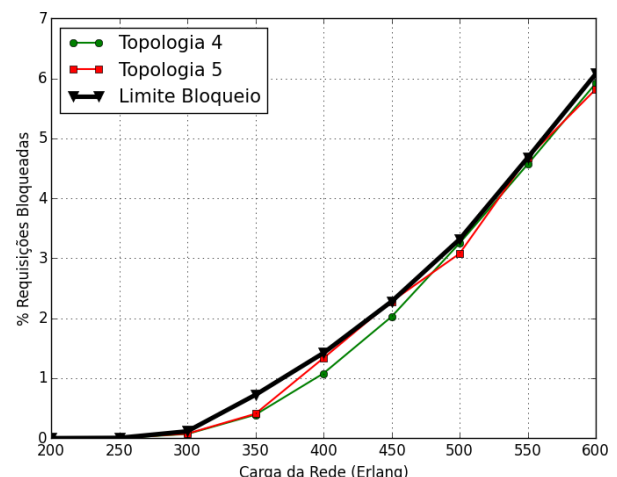

(b)

Figura 4. (a) Curva de bloqueio permitido, utilizando percentil de $80 \%$. (b) Curvas resultantes após aplicação do filtro de limite de bloqueio máximo permitido e a curva do bloqueio permitido.

da rede no intervalo analisado. Os métodos avaliam as curvas de forma independente dentro do intervalo analisado. Um faz a comparação entre as curvas e o limite de bloqueio para cada carga da rede, e o outro calcula a área sob a curva, resultando em um valor global para cada topologia que representa o seu desempenho.

\subsection{Método de avaliação considerando as cargas da rede (MCR)}

O método permite a atribuição de um valor de importância para cada carga de rede, podendo dar maior importância para as cargas mais baixas ou mais altas. Nesse caso é considerado que conforme a carga da rede aumenta, a taxa de bloqueio também aumenta devido à concorrência por recursos gerada pelas requisições, e o método considera mais importante as cargas mais baixas, onde a concorrência por recursos é menor e a rede não está sobrecarregada.

O valor de importância de uma carga $I_{n}$ é calculado a partir da expressão:

$$
I_{n}=N-\left(V * P_{n}\right)
$$

onde $N$ é o valor de importância inicial, $V$ é um peso que contribui para a distinção da importância e $P_{n}$ é o índice da enésima carga, iniciando em 0 (zero), sendo que $P_{0}=$ $0, P_{1}=1, \ldots, P_{n}=N$. Quanto maior o valor dado a $N$ maior será a importância para as cargas mais baixas (com índice menor) e quanto maior o valor dado a $V$, maior será a diferença de importância entre as cargas no intervalo analisado.

A Figura 5 ilustra o exemplo de cálculo considerando a carga de 400 Erlang, ou seja, $I_{4}$ e $P_{4}=4$. Inicialmente toma-se o valor do bloqueio de uma topologia e calculase o módulo da diferença deste valor com o valor do bloqueio das outras topologias com maior bloqueio. Note que a topologia 4 apresenta bloqueio inferior à topologia 5 e inferior à curva de limite de bloqueio, logo é preciso calcular duas diferenças. Note também que, para a topologia 5, o bloqueio é inferior apenas à curva de limite de bloqueio, logo é preciso calcular apenas uma diferença.

Para cada topologia $t$ um valor será calculado a partir da expressão:

$$
\text { Ponto }\left[P_{n}\right]_{t}=\sum_{i=1}^{m} \text { Diferenca }_{t, i} * \exp \left(N-\left(V * P_{n}\right)\right)
$$




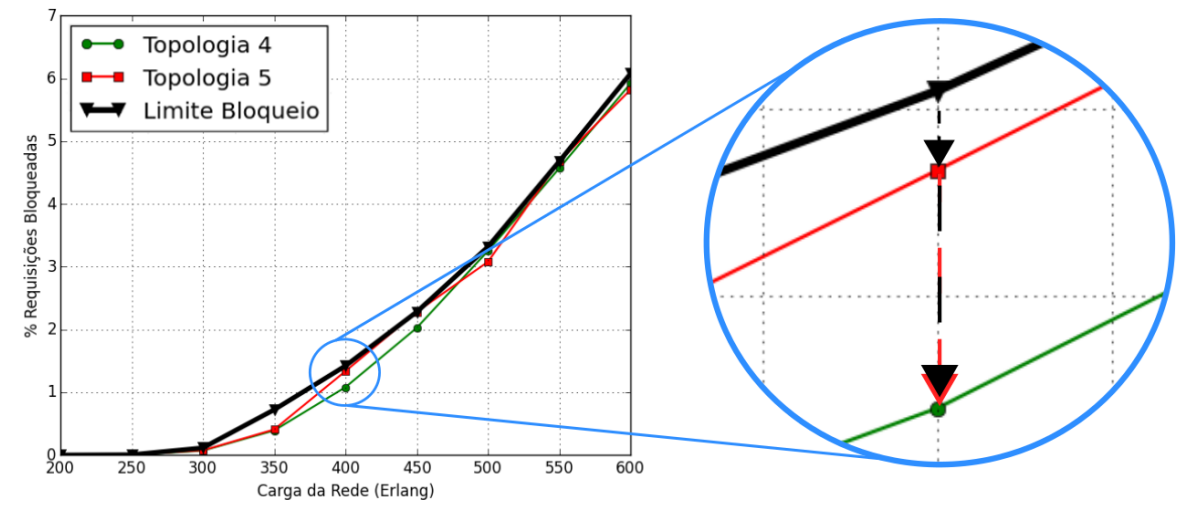

Figura 5. Zoom aplicado na carga da rede com 400 Erlangs para demonstração de como é computada a diferença entre os valores de bloqueio entre as topologias.

onde $m$ é o número de topologias (incluindo a curva de limite máximo) com bloqueio maior que a topologia $t$, Diferenca $a_{t, i}$ é o módulo da diferença entre bloqueio de $t$ e o bloqueio da topologia $i$, sendo a topologia $i$ aquela com bloqueio maior que $t$ em $P_{n}$.

Utilizando como exemplo os valores de $N=10$ e o $V=1$ e a topologia 4, conclui-se que a mesma possui a taxa de bloqueio inferior a topologia 5 e ao limite máximo permitido para a carga da rede 400. Logo serão computadas 2 diferenças, a Diferenca 1 que será a diferença entre os valores de taxa de bloqueio das topologias $4 \mathrm{e}$ 5 , e a Diferenca 2 que será a diferença entre os valores de limite máximo de bloqueio e a taxa de bloqueio da topologia 4. Para a topologia 4 o valor é calculado da seguinte forma:

$$
\text { Ponto }[4]_{4}=\text { Diferenca } 1 * \exp (10-(1 * 4))+\text { Diferenca } 2 * \exp (10-(1 * 4))
$$

compondo assim o valor para a topologia 4 no ponto $P_{4}=4$. Para a topologia 5 , só existirá uma diferença que é calculada através da diferença do limite máximo de bloqueio e o valor referente ao bloqueio da topologia 5. O cálculo é feito para todas as cargas no intervalo analisado e, no final do processo, é feito um somatório para cada topologia. Aquela que possuir o maior valor resultante do somatório será considerada a topologia com melhor desempenho em um contexto global.

Para o caso das topologias classificadas apresentadas na Figura 4 (b), a que possui o melhor desempenho, conforme o método, é a 4. Os resultados mostram que, em algumas cargas, a taxa de bloqueio da topologia 5 é igual ao limite máximo de bloqueio permitido. Nessas condições não se agrega valor para as cargas. Ainda, na maioria das cargas, principalmente as mais baixas, a topologia 5 apresentou bloqueios iguais ou superiores à topologia 4.

\subsection{Método de avaliação considerando a área sob a curva (MAC)}

O método calcula área sob a curva dentro do intervalo analisado, e cada curva é analisada individualmente sem efeitos das demais, possuindo menor complexidade quando comparado ao método anterior. O MAC considera que todas as cargas da rede têm a mesma importância, e são analisadas da mesma forma.

Na Figura 6 são mostradas as áreas sob as curvas das topologias 4 e 5 apresentadas na Figura 4 (b). Das topologias avaliadas, este método identifica a topologia 4 como a 
que possui o melhor desempenho, por possuir uma curva de bloqueio cuja a área é menor que a área sob a curva de bloqueio da topologia 5.

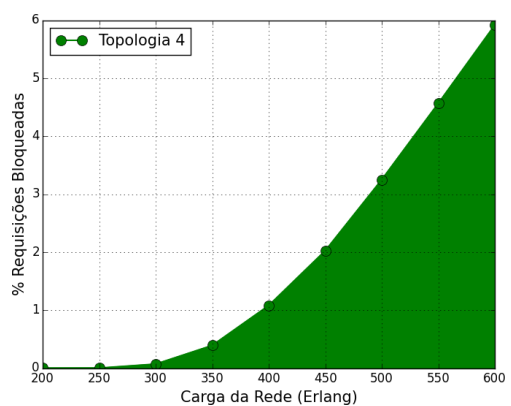

(a)

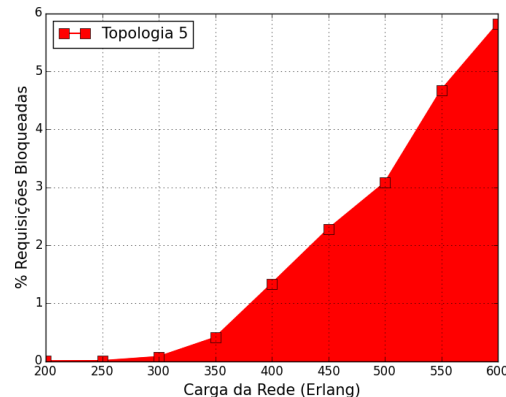

(b)

Figura 6. (a) Área sob a curva de bloqueio da Topologia 4. (b) Área sob a curva de bloqueio da Topologia 5.

\subsection{Comparação e análise dos métodos}

Ambos os métodos indicam uma topologia com o melhor desempenho em um contexto global, porém os resultados da avaliação podem alterar conforme o método. A principal diferença entre os métodos é a forma como abordam as taxas de bloqueio conforme a mudança das cargas da rede.

A Figura 7 mostra duas curvas de bloqueio, onde não é possível identificar visualmente qual apresenta o melhor resultado global. Ao comparar as duas curvas utilizando os métodos propostos, o MCR considera a Curva 2 como melhor, pois os resultados das cargas mais baixas possuem mais importância e possuem a taxa de bloqueio menor que a Curva 1. Para o MAC a Curva 1 é avaliada como melhor, pois as cargas de rede não possuem um valor de importância associado a elas, e a alteração nas cargas de rede mais altas impactam mais a área sob a curva do que as alterações das cargas mais baixas.

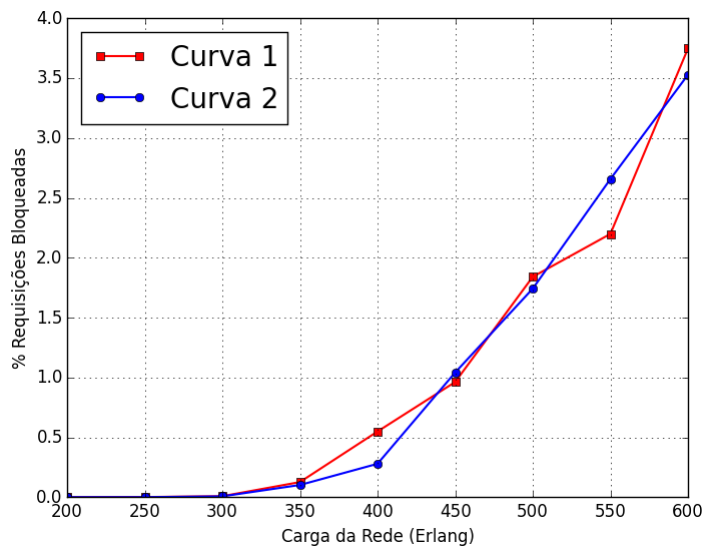

Figura 7. Curva 1 melhor para MAC e Curva 2 melhor para MCR.

Considerando que o bloqueio acontecerá com maior frequência quando a rede está sobrecarregada, o MCR se mostra mais adequado ao cenário em estudo. Porém, a análise deve ser feita em comparação com as demais curvas, não sendo possível obter um resultado definitivo avaliando a curva individualmente, como no caso do MAC onde os resultados podem ser avaliados separadamente. 


\section{Resultados}

Como forma de avaliação dos métodos propostos, são considerados conjuntos de topologias que possuem a mesma quantidade de nós e enlaces, geradas a partir da adição de um único enlace nas redes RNP e NSFNET, gerando todas as combinações com o mesmo número de nós e o número de enlaces +1 . A topologia original da RNP possui 22 nós e 31 enlaces, e com a adição de uma aresta, obtivemos um conjunto de 200 topologias com 22 nós e 32 enlaces. Para a rede NSFNET de 14 nós e 21 enlaces, obtivemos um conjunto de 70 topologias de 14 nós e 22 enlaces. Para cada conjunto, o método deve indicar quais são as topologias que possuem o melhor desempenho, considerando os resultados da taxa de bloqueio de cada topologia.

Para a aplicação do RSA nas topologias geradas e na topologia original, foi utilizada a ferramenta de simulação de redes ópticas elásticas ElasticO++ [Tessinari et al. 2016] [Neris et al. 2019]. Para o estudo proposto, foram configurados 360 slots $(360 \cdot 12,5 \mathrm{GHz}=4,5 \mathrm{THz})$ por fibra, para cada simulação foram aplicadas 200000 requisições, e para cada carga da rede foram aplicadas 5 simulações para cada rede, com 5 sementes randômicas diferentes. As requisições demandam 4 tipos de tráfego, sendo eles de 40,100, 400 e $1000 \mathrm{~Gb} / \mathrm{s}$, com um padrão de tráfego de 4:3:2:1 respectivamente. O algoritmo de RSA utilizado foi o Algo-1 [Tessinari et al. 2016], que utiliza o algoritmo de roteamento Dijkstra, o formato de modulação EON DP-QPSK, a técnica Spectrum Sharing para gerenciamento do espectro e First-Fit para a alocação do espectro. Todas as técnicas utilizadas já estão implementadas na ferramenta ElasticO++.

Após a obtenção dos resultados através da simulação do RSA, foram aplicados três filtros de limite de bloqueio utilizando o cálculo de percentil para os valores de $100 \%$, $50 \%$ e 20\%. Para o MCR, além do valor de percentil (P) existem os valores de importância inicial $(\mathrm{N})$ e o valor de variação $(\mathrm{V})$, e na Tabela 1 são ilustradas as 6 configurações utilizadas. Para o MAC não existem parâmetros adicionais, sendo considerados apenas os valores do cálculo de percentil.

Tabela 1. Configurações utilizadas para o teste do MCR.

\begin{tabular}{c|c|c|c|c|c|c}
\hline & \multicolumn{6}{|c}{ Configurações } \\
\hline Parâmetros & $\mathbf{1}$ & $\mathbf{2}$ & $\mathbf{3}$ & $\mathbf{4}$ & $\mathbf{5}$ & $\mathbf{6}$ \\
\hline$P$ & $100 \%$ & $100 \%$ & $50 \%$ & $50 \%$ & $20 \%$ & $20 \%$ \\
$N$ & 1 & 10 & 1 & 10 & 1 & 10 \\
$V$ & 0.1 & 1 & 0.1 & 1 & 0.1 & 1 \\
\hline
\end{tabular}

Os nomes das topologias possuem o formato $i_{-} j$, onde $i$ e $j$ representam os nós da topologia e $i_{-} j$ representa a aresta que foi adicionada a partir da topologia original.

Para a rede RNP foram testadas 201 topologias diferentes, sendo 200 oriundas da adição de um enlace que não existe na rede original mais a rede original. Na Tabela 2 são apresentados os resultados para as 6 configurações aplicadas para o MCR, onde é possível ver quais as 3 melhores topologias e a quantidade de topologias classificadas após a aplicação do filtro.

Na Tabela 3 são apresentados os resultados para a rede RNP para o MAC, onde 
Tabela 2. Resultados da rede RNP para a aplicação do MCR.

\begin{tabular}{c|c|c}
\hline Configuração & Melhores Topologias & \# Top. Classificadas \\
\hline 1 & $2 \_17,7 \_17,2 \_16$ & 201 \\
2 & $2 \_17,7 \_17,2 \_16$ & 201 \\
3 & $2 \_17,7 \_17,2 \_16$ & 45 \\
4 & $2 \_17,7 \_17,2 \_16$ & 45 \\
5 & $2 \_17,7 \_17,2 \_16$ & 11 \\
6 & $2 \_17,7 \_17,2 \_16$ & 11 \\
\hline
\end{tabular}

é possível observar que as melhores topologias se mantiveram as mesmas para ambos os métodos, para todas as configurações apresentadas nas Tabelas 2 e 3. Na configuração com $P=20 \%, 11$ das 201 topologias não tiveram um bloqueio maior que o limite de bloqueio máximo permitido. É interessante salientar que mesmo com uma aresta a menos, a topologia original da RNP obtém um bloqueio menor que outras topologias, como mostra a Figura 8 (b), possuindo melhor desempenho que 17 topologias para o MCR e 16 topologias para o MAC, significando que adicionar novos enlaces pode piorar o desempenho da rede quando mantido o mesmo método de roteamento e alocação de espectro.

Tabela 3. Resultados da rede RNP para a aplicação do MAC.

\begin{tabular}{c|c|c}
\hline Percentil & Melhores Topologias & \# Top. Classificadas \\
\hline $100 \%$ & $2 \_17,7 \_17,2 \_16$ & 201 \\
$50 \%$ & $2 \_17,7 \_17,2 \_16$ & 45 \\
$20 \%$ & $2 \_17,7 \_17,2 \_16$ & 11 \\
\hline
\end{tabular}

Na Figura 8 (a) tem-se a representação das curvas de bloqueio referentes a topologia RNP, das 3 melhores topologias $\left(2 \_17,7 \_17\right.$ e $2 \_16$ os enlaces podem ser visualizados na Figura 10 (a)) encontradas pelo método, a curva de bloqueio da topologia original, e as curvas de limite máximo de bloqueio permitido de $50 \%$ e $20 \%$, dentre todas as cargas, a variância máxima das curvas foi de $0,5 \%$ nas cargas de 600 Erlangs.

Para a rede NSFNET foram testadas 71 topologias diferentes, das quais, 70 surgiram da adição de um enlace à rede original e uma é a própria rede NSFNET. Na Tabela 4 tem-se os resultados para as 6 configurações para o MCR.

Tabela 4. Resultados da rede NSFNET para a aplicação do MCR.

\begin{tabular}{c|c|c}
\hline Configuração & Melhores Topologias & \# Top. Classificadas \\
\hline 1 & $4 \_8,2 \_12,3 \_12$ & 71 \\
2 & $4 \_8,2 \_12,3 \_12$ & 71 \\
3 & $4 \_8,3 \_12,1 \_12$ & 17 \\
4 & $4 \_8,3 \_12,1 \_12$ & 17 \\
5 & $1 \_12$ & 1 \\
6 & $1 \_12$ & 1 \\
\hline
\end{tabular}




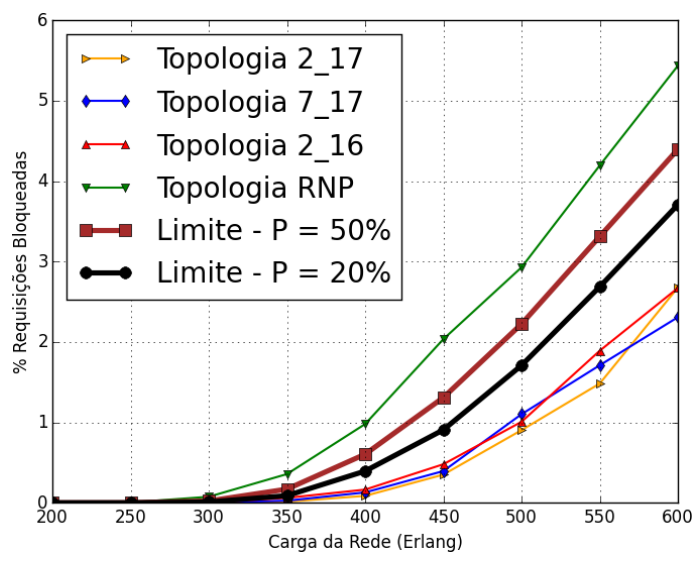

(a)

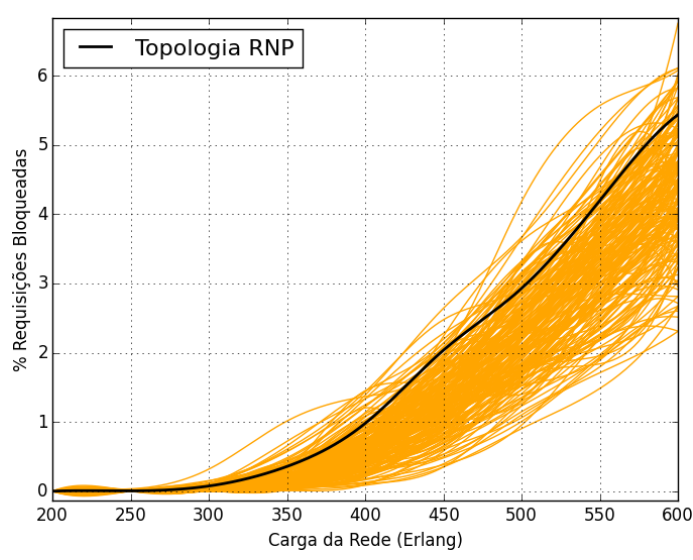

(b)

Figura 8. (a)Curvas de bloqueio e de Limite máximo permitido para a rede RNP. (b) Curvas de bloqueio das 201 topologias, destacando a curva da Topologia RNP original.

Na Tabela 5 são apresentados os resultados para a rede NSFNET utilizando o MAC. Diferentemente dos resultados obtidos com as topologias originadas da RNP, as melhores topologias originadas da NSFNET se alteram conforme as configurações e os métodos, conforme apresentado nas Tabelas 4 e 5. Nas configurações com $P=20 \%$, somente a topologia 1_12 não teve um bloqueio maior que o limite de bloqueio máximo permitido. Na avaliação com $P=100 \%$ a topologia $1 \_12$ é a $5^{a}$ melhor topologia para o MCR e a $4^{a}$ melhor para o MAC. Na Figura 9 (c) são apresentadas as curvas de bloqueio das 71 topologias, onde é possível observar que existem topologias que apresentam um pior desempenho que a topologia original, reforçando que adicionar um enlace extra pode, contraintuitivamente, piorar o desempenho da rede.

Tabela 5. Resultados da rede NSFNET para a aplicação do MAC.

\begin{tabular}{c|c|c}
\hline Percentil & Melhores Topologias & \# Top. Classificadas \\
\hline $100 \%$ & $4 \_8,3 \_12,2 \_12$ & 71 \\
$50 \%$ & $4 \_8,3 \_12,1 \_12$ & 17 \\
$20 \%$ & $1 \_12$ & 1 \\
\hline
\end{tabular}

A Figura 9 (a) apresenta as curvas de bloqueio das topologias que estiveram entre as topologias com o melhor desempenho em alguma das configurações testadas (4_8, $2 \_12,3 \_12$ e 1_12. Os enlaces podem ser visualizados na Figura 10), incluindo a própria rede NSFNET, dentre todas as cargas, a variância máxima das curvas foi de 0,3\% nas cargas de 600 Erlangs. É possível identificar que as topologias 4_8 e 3_12 apresentam bloqueio maior que a curva de limite de $P=20 \%$ nas cargas 500 e 600 , respectivamente. A topologia 2_12 apresenta bloqueio maior que a curva de limite de $P=50 \%$ na carga 300, sendo possível observar na Figura 9 (b), onde foi aplicado um zoom no ponto 300 do gráfico da Figura 9 (a).

Na Figura 10 tem-se as topologias RNP (a) e NSFNET (b), com a indicação dos enlaces adicionais que aumentaram o desempenho da rede em uma análise global, con- 


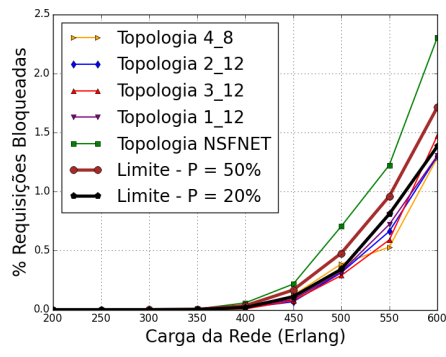

(a)

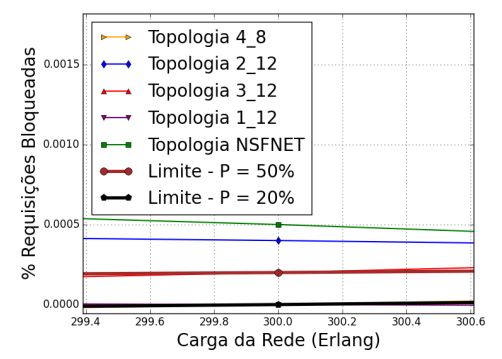

(b)

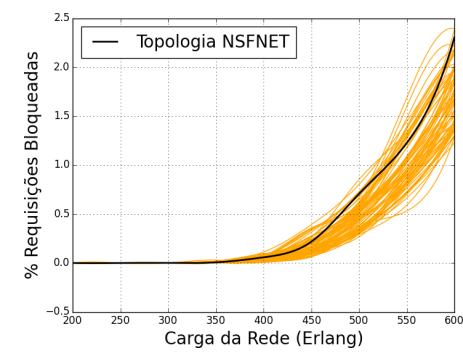

(c)

Figura 9. (a) Curvas de bloqueio e curvas de Limite máximo permitido para a rede NSFNET. (b) Zoom aplicado na carga da rede 300 da Figura 9 (a). (c) Curvas de bloqueio das 71 topologias, destacando a curva da Topologia NSFNET original.

forme o método proposto. Observando que a adição de um enlace gera uma nova topologia, para a rede RNP da Figura 10 (a) existem 3 topologias diferentes mais a original e na rede NSFNET da Figura 10 (b) os 4 enlaces adicionais geram 4 novas topologias.

\subsection{Análise dos Resultados}

As simulações revelaram que a carga de rede afeta o desempenho de uma topologia de rede. Comparar duas topologias com uma mesma carga de tráfego não permite identificar a topologia com melhor desempenho global, ou seja, para diversas cargas de tráfego.

Para a rede RNP as melhores topologias se mantiveram para todas as configurações e para os dois métodos. Porém, para a rede NSFNET, as melhores topologias foram desclassificadas quando diminuído o valor do percentil $P$, mostrando que, para algumas cargas, elas não estiveram entre as melhores. Este pode ser um fator importante para a escolha da melhor topologia consoante o cenário apresentado.

Para os testes realizados foi adotada a topologia 1_12 como a melhor topologia para a rede NSFNET pois ela é a única que está entre as $20 \%$ com o melhor desempenho em todas as cargas de rede analisadas. Porém, para a rede RNP, as topologias 2_17, 7_17 e 2_16 tiveram resultados satisfatórios e as 3 são consideradas topologias que possuem um bom desempenho para os testes, pois todas possuem um comportamento estável, se mantendo entre as topologias que tem o melhor desempenho, em todos os testes realizados utilizando os métodos apresentados.

Com a adição de um enlace na topologia, foi possível perceber a alteração do desempenho da rede. Na maioria dos casos o efeito foi positivo, mas, houve casos em que aumentou a taxa de bloqueio.

\section{Conclusão e Trabalhos Futuros}

Dois métodos para a análise de curvas de bloqueio foram propostos, com vista a identificar topologias de rede com melhor desempenho global em relação à taxa de bloqueio. Os métodos facilitam a identificação dos melhores resultados apresentados por curvas de bloqueio e podem ser utilizados em conjunto com outras métricas.

Foram apresentados os resultados obtidos pela aplicação do RSA a dois conjuntos de topologias, onde foi possível confirmar (em comparação com os resultados demons- 


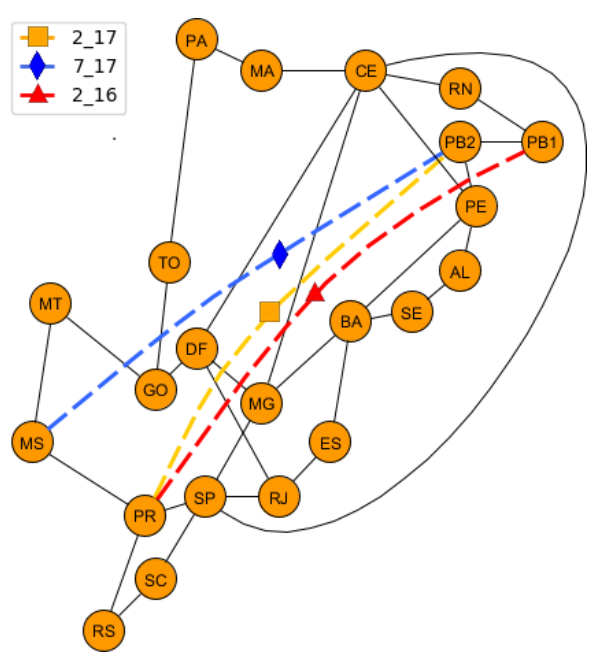

(a)

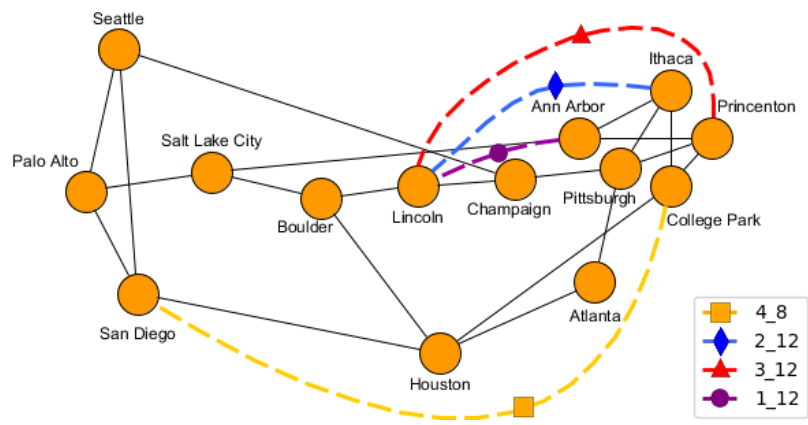

(b)

Figura 10. (a) Rede RNP com os enlaces adicionais que geraram as topologias 2_17, 7_17 e 2_16, identificadas como as que possuem melhor desempenho para os métodos implementados. (b) Rede NSFNET com enlaces adicionais que geraram as topologias $4 \_8,2 \_12,3 \_12$ e 1_12, identificadas como as que possuem melhor desempenho para os métodos implementados.

trados em [Tessinari et al. 2018b]) que topologias com a mesma quantidade de recursos podem possuir taxas de bloqueio diferentes, e topologias com menos recursos ( 1 enlace a menos) podem possuir menor taxa de bloqueio do que topologias com mais recursos disponíveis para a alocação das demandas. Ambos os métodos foram capazes de classificar as topologias conforme o seu desempenho em todas as cargas do intervalo analisado, e mesmo tendo abordagens diferentes classificaram as mesmas topologias como as melhores, atendendo o limiar especificado. Porém, existem casos onde pode retornar um subconjunto de topologias ou até mesmo nenhuma topologia.

Em trabalhos futuros a sugestão é utilizar os métodos propostos para avaliar, de forma sistemática, os resultados de bloqueio obtidos através da aplicação de algoritmos de roteamento e alocação, e da alteração das características da rede. E assim, auxiliar na tomada de decisões e implementação de heurísticas capazes de diminuir o bloqueio da rede em uma análise global.

\section{Agradecimentos}

Este trabalho foi parcialmente apoiado financeiramente pela Fundação de Amparo à Pesquisa e Inovação do Espírito Santo (FAPES) e pelo Conselho Nacional de Desenvolvimento Científico e Tecnológico (CNPq), através do projeto CAGETOTE - CAracterização e GEração de TOpologias físicas para redes de transporte de TElecomunicações, processo n. 406924/2016-3.

\section{Referências}

(2015). Rede nacional de ensino e pesquisa. https : / / www . rnp. br/node/735. 06 fev. 2020. 
Chatterjee, B. C., Ba, S., and Oki, E. (2018). Fragmentation problems and management approaches in elastic optical networks: A survey. IEEE Communications Surveys \& Tutorials (Volume: 20, Issue: 1, Firstquarter 2018), pages 183-210.

Chatterjee, B. C., Nityananda, S., and Oki, E. (2015). Routing and spectrum allocation in elastic optical networks: A tutorial. IEEE Communications Surveys \& Tutorials (Volume: 17, Issue: 3, thirdquarter 2015). pp 1776 - 1800.

Dijkstra, E. W. (1959). A note on two problems in connexion with graphs. Numer. Math., 1 (1) (1959), pp. 269-271, Google Scholar.

Gerstel, O., Jinno, M., Lord, A., and Yoo, S. J. B. (2012). Elastic optical networking: A new dawn for the optical layer? IEEE Communications Magazine (Volume: 50, Issue: 2, February 2012), pages s12-s20.

Neris, Y. G., Tessinari, R. S., Garcia, A. S., Segatto, M. E. V., and Paiva, M. H. M. (2019). Enabling user-friendly eon simulations with a graphical interface for elastico++. In 2019 SBMO/IEEE MTT-S International Microwave \& Optoelectronics Conference (IMOC). IEEE.

Pavan, C., de Lima, L. S., Paiva, M. H. M., and Segatto, M. E. V. (2015). How reliable are the real-world optical transport networks? IEEE/OSA Journal of Optical Communications and Networking, 7(6):578-585.

Ramamurthy, R. and Mukherjee, B. (2002). Fixed-alternate routing and wavelength conversion in wavelength-routed optical networks. IEEE/ACM Transactions on Networking (Volume: 10 , Issue: 3 , Jun 2002), pages 351-367.

Rosa, A., Cavdar, C., Carvalho, S., Costa, J., and Wosinska, L. (2012). Spectrum allocation policy modeling for elastic optical networks. High Capacity Optical Networks and Emerging/Enabling Technologies.

Tessinari, R. S., Colle, D., and Garcia, A. S. (2018a). Cognitive zone-based spectrum assignment algorithm for elastic optical networks. In 2018 International Conference on Optical Network Design and Modeling (ONDM), pages 112-117. IEEE.

Tessinari, R. S., Paiva, M. H. M., Monteiro, M. E., Segatto, M. E. V., Garcia, A. S., Kanellos, G. T., Nejabati, R., and Simeonidou, D. (2018b). On the impact of the physical topology on the optical network performance. In 2018 IEEE British and Irish Conference on Optics and Photonics (BICOP), pages 1-4. IEEE.

Tessinari, R. S., Puype, B., Colle, D., and Garcia, A. S. (2015). Zone based spectrum assignment in elastic optical networks: A fairness approach. In 2015 Opto-Electronics and Communications Conference (OECC), pages 1-3. IEEE.

Tessinari, R. S., Puype, B., Colle, D., and Garcia, A. S. (2016). Elastico++: An elastic optical network simulation framework for omnet++. Optical Switching and Networking, 22:95-104.

Wang, Y., Cao, X., and Pan, Y. (2011). A study of the routing and spectrum allocation in spectrum-sliced elastic optical path networks. 2011 Proceedings IEEE INFOCOM.

Zang, H., Jue, J. P., Mukherjee, B., et al. (2000). A review of routing and wavelength assignment approaches for wavelength-routed optical wdm networks. Optical networks magazine, 1(1):47-60. 